\title{
The Application of a New Neutron Induced Gamma Ray Spectroscopy Tool in Evaluation the Shale Gas in Fuling Shale Gas Field
}

\author{
Wei Yan ${ }^{*}$, Minggang Feng1, Yue Wang2, Shuai Liu1, Kaixuan Li², Xianran Zhao², Wenjun Liang ${ }^{3}$ \\ ${ }^{1}$ Sinopec Exploration Company, Chengdu, China \\ ${ }^{2}$ Schlumberger China S.A., Chengdu, China \\ ${ }^{3}$ Exploration and Development Research Institute of Huabei Oil Field Company, PetroChina, Renqiu, China \\ Email: *yanweimako@126.com
}

How to cite this paper: Yan, W., Feng, M.G., Wang, Y., Liu, S., Li, K.X., Zhao, X.R. and Liang, W.J. (2018) The Application of a New Neutron Induced Gamma Ray Spectroscopy Tool in Evaluation the Shale Gas in Fuling Shale Gas Field. Open Journal of Yangtze Gas and Oil, 3, 93-103. https://doi.org/10.4236/ojogas.2018.32008

Received: August 17, 2017

Accepted: April 25, 2018

Published: April 28, 2018

Copyright ( 92018 by authors and Scientific Research Publishing Inc. This work is licensed under the Creative Commons Attribution International License (CC BY 4.0).

http://creativecommons.org/licenses/by/4.0/

\section{(c) (i) Open Access}

\begin{abstract}
Shale gas reservoir is characterized by complex lithology with ultra-low porosity and permeability, which brings many challenges in the formation evaluation. Elemental Capture Spectroscopy Sonde (ECS) can be used to measure the elements and get the formation of the mineralogy. LithoScanner is a newly-generated spectroscopy tool, which can be used to measure both inelastic and capture spectra, providing estimation of more elements, including silicon, calcium, iron, sulfur, titanium, gadolinium, aluminum, potassium, sodium, magnesium, manganese, carbon, etc. LithoScanner can be directly used to measure the total organic carbon content (TOC) in the formation, and provide solutions to the lithology classification and heterogeneous rock analysis (HRA). The changing lithology and borehole rugosity have little influence on the direct measurement of TOC by LithoScanner. LithoScanner shows great advantage in accuracy when comparing with other methods using triple-combo. The systematic lithology classification method in shale gas reservoir using LithoScanner is correlatable in multi-wells. The brittleness index from mineralogy can be applied to hydraulic stimulation. HRA results offered guides in both the coring sample selection and the experiment. The advantages of LithoScanner in shale gas formation evaluation were presented in this paper, and the workflow can also be applied to other reservoirs.
\end{abstract}

\section{Keywords}

LithoScanner, ECS, TOC, Lithology Classification, HRA, Fuling Shale Gas

\section{Introduction to LithoScanner Tool}

LithoScanner is an innovation of gamma ray spectroscopy tool, and is capable of 
measuring accurate mineralogy [1]. Elements that can be measured from capture and inelastic gamma ray spectroscopy of LithoScanner are shown in Table 1. LithoScanner can not only be used to accurately measure the elements in the formation, but it can also be used to output the total organic carbon content (TOC) of the formation. Figure 1 is the sketch map of the LithoScanner tool, whose outer diameter is 4.5 in, maximum pressure is 20,000 psi, maximum temperature is $175^{\circ} \mathrm{C}$, and vertical resolution is $18 \mathrm{in}$.

Table 1. Elements measured in inelastic and capture spectroscopy using LithoScanner and ECS.

\begin{tabular}{|c|c|c|c|}
\hline \multirow{2}{*}{ Element } & \multicolumn{2}{|c|}{ LithoScanner } & \multirow{2}{*}{$\begin{array}{c}\text { ECS } \\
\text { Capture }\end{array}$} \\
\hline & Inelastic & Capture & \\
\hline $\mathrm{Al}$ & $\sqrt{ }$ & $\sqrt{ }$ & $\sqrt{ }$ (Indirectly) \\
\hline $\mathrm{Ba}$ & $\sqrt{ }$ & $\sqrt{ }$ & \\
\hline $\mathrm{C}$ & & $\sqrt{ }$ & \\
\hline $\mathrm{Ca}$ & $\sqrt{ }$ & $\sqrt{ }$ & $\sqrt{ }$ \\
\hline $\mathrm{Cl}$ & $\sqrt{ }$ & & \\
\hline $\mathrm{Cu}$ & $\sqrt{ }$ & & \\
\hline $\mathrm{Fe}$ & $\sqrt{ }$ & $\sqrt{ }$ & $\sqrt{ }$ \\
\hline Gd & $\sqrt{ }$ & & $\sqrt{ }$ \\
\hline $\mathrm{H}$ & $\sqrt{ }$ & & \\
\hline $\mathrm{K}$ & $\sqrt{ }$ & & \\
\hline $\mathrm{Mg}$ & $\sqrt{ }$ & $\sqrt{ }$ & \\
\hline $\mathrm{Mn}$ & $\sqrt{ }$ & & \\
\hline $\mathrm{Na}$ & $\sqrt{ }$ & & \\
\hline $\mathrm{Ni}$ & $\sqrt{ }$ & & \\
\hline $\mathrm{O}$ & & $\sqrt{ }$ & \\
\hline S & $\sqrt{ }$ & $\sqrt{ }$ & $\sqrt{ }$ \\
\hline $\mathrm{Si}$ & $\sqrt{ }$ & $\sqrt{ }$ & $\sqrt{ }$ \\
\hline $\mathrm{Ti}$ & $\sqrt{ }$ & & $\sqrt{ }$ \\
\hline
\end{tabular}

\subsection{Data Acquisition and Processing}

The data acquisition and processing of LithoScanner are as follows.

1) Gamma ray is induced after the fast neutron interacts with the elements in the formation. There are two major benefits of the well-defined neutron burst. The first one is that there is no contamination by inelastic reactions during the "capture" gates. The other one is that the capture data can be collected very soon after the burst.

2) Gamma ray spectra to the yields processing. Capture and inelastic gamma ray spectra are measured simultaneously, and the relative yields of elements from both spectra are calculated independently. Elemental relative yields are a 

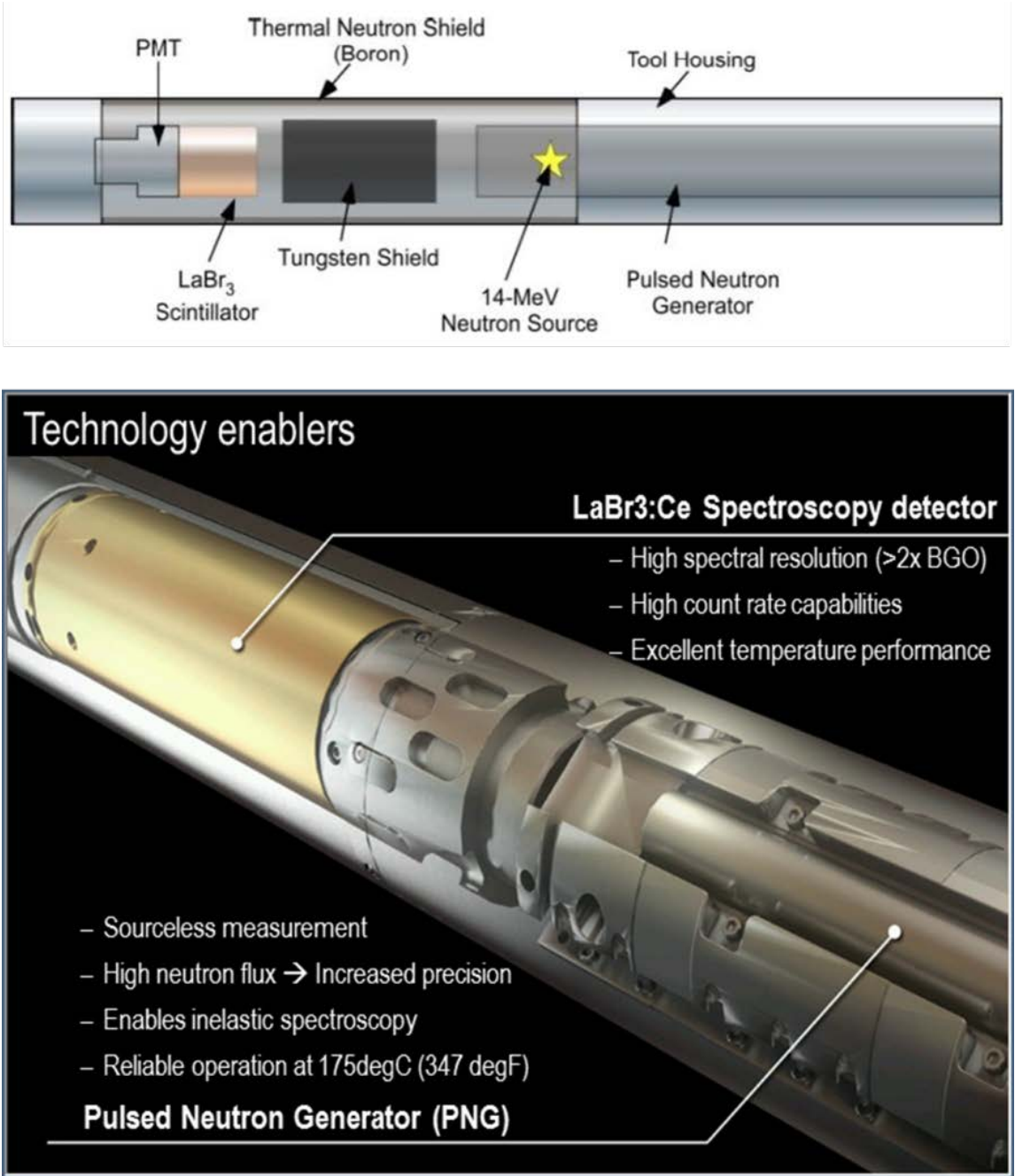

Figure 1. Sketch map of LithoScanner.

function of the volumetric proportion of an element in the measurement region, as well as the sensitivity of the tool to each element.

3) Yields to dry weight elements. The conversion of relative spectral yields from neutron capture into absolute elemental concentrations is accomplished via a modified geochemical oxides closure model, which is similar to the ECS. An important limitation of the inelastic physics is that its set of yields is not complete enough to form a closure model. Instead, the normalization factor that converts these inelastic yields into elemental concentrations is derived by the derived dry weights of some of the elements, which occur in both inelastic and capture measurements and are consistent. The method ensures that all the resulting dry weights are mutually consistent for elements with both capture and inelastic yields. As a consequence, the analysis gains the enhanced precision of the inelastic Mg measurement and achieves a better accuracy overall.

4) Dry weight elements to minerals. The mineralogy can be derived from the elements using an inversion method. The quantitative elemental concentrations and processed lithology can be used directly with other log data in petrophysical 
interpretation programs to provide more accurate petrophysical answers.

\subsection{The Advantages of LithoScanner}

1) New spectroscopy detector. The tool uses a large cerium-doped lanthanum bromide $(\mathrm{LaBr} 3: \mathrm{Ce})$ gamma ray detector, which is coupled to a high-temperature photomultiplier. LaBr3:Ce is a very fast scintillator with high light output and excellent spectral resolution. In addition, $\mathrm{LaBr} 3$ :Ce has an outstanding high-temperature performance with only a minimal loss in light output and resolution at temperatures up to $200^{\circ} \mathrm{C}$.

2) New pulsed neutron generator (PNG). Instead of using chemical source $241 \mathrm{AmBe}$, LithoScanner is equipped with PNG, which emits neutron into the formation to facilitate the measurement of spectroscopy. The new measurement is sourceless, and has a better performance.

3) The advantage of LithoScanner over ECS is that LithoScanner can be used to measure both capture and inelastic gamma ray spectroscopy, with an increase in the measured elements (Table 1), while ECS can only be used to measure capture gamma ray spectroscopy [2] [3] [4] [5]. The mineralogy derived from spectroscopy can be subdivided into 7 categories (clay, quartz, k-feldspar, Na-feldspar, calcite, dolomite, pyrite) in LithoScanner, comparing to 4 categories (clay, quartz-feldspar-mica, carbonate, pyrite) in ECS. The clay can be further elaborated into illite and chlorite.

4) LithoScanner can be used to measure the TOC in the formation. Total in-organic carbon (TIC) can be computed from the carbon in carbonate, which derives from LithoScanner spectroscopy results. TOC is then calculated by subtracting TIC from the total carbon measured by LithoScanner.

5) Heterogeneity rock analysis (HRA) can be done using the combination of elements from LithoScanner and triple-combos. Rocks with identical petrophysical properties can be classified into the same category. This robust and handy method can be a guide for the coring, and improve the efficiency.

\subsection{The Contribution of LithoScanner to Shale Gas Formation Evaluation}

TOC is one of the most important reservoir quality parameters in shale gas formation evaluation. A lot of literatures have use it for reservoir classification [6] [7] [8] [9] [10], and the TOC is usually relied on core experiments. However, the long period of the core experiments is a headache for the geologists. Apart from core experiments, the TOC can also be calculated via triple-combos, such as density, GR, etc. [11]. The drawback of this methodology is that it requires core data for calibration. All the petrophysical results will be updated if the core TOC and petrophyscis derived TOC don't match. What is more, core experiments are usually time-consuming and thus un-acceptable since the petroleum exploration is now in fast pace.

As the first application of LithoScanner in Fuling shale gas field, this paper has 
demonstrated the great success in the application. The LithoScanner derived TOC matches well with the mud log TOC. It proves the robustness of this tool and methodology. It is also proposed to log LithoScanner in the later shale gas exploration wells in SinoPec.

\section{Data Interpretation}

\subsection{Computation of TOC}

A lot of works have been done in sedimentology, reservoir quality, preservation of Silurian Longmaxi shale gas reservoir in Sichuan basin and its surrounding area in China recently [6] [7] [8] [9] [10]. TOC is a brief of total organic carbon content, and is an important parameter in petrophysical analysis of shale gas reservoir. The routine method for TOC is indirect, which requires the linear fitting of core TOC and porosity logs. There are some drawbacks of this TOC computation methodology, for example, bulk density will be lowered due to the washout in the borehole, which will lower the accuracy of TOC computation methodology. The linear fitting of core TOC and porosity logs requires a large number of core inputs, and the function will not be applied to all the areas [11] [12]. The advantage of LithoScanner is that common association factors can be used for carbonate minerals. TIC can be computed from the carbon in carbonate, which derives from LithoScanner spectroscopy results. TOC is then calculated by subtracting TIC from the total carbon measured by LithoScanner. The methodology is independent of the borehole rougosity, and has good applications in many shale reservoirs globally [13] [14] [15].

The application of LithoScanner in well A of Jiaoshiba shale gas field in Fuling was discussed in this paper.

In Figure 2, the $1^{\text {st }}$ track is measured depth and total gas from mud log, the $2^{\text {nd }}$ track is GR, uranium free GR, and caliper; the $3^{\text {rd }}$ track is the deep and shallow laterolog resistivities; the $4^{\text {th }}$ track is neutron, density, DT and PEF; the $5^{\text {th }}$ track is thorium, uranium and potassium; the $6^{\text {th }}$ track is TOC derived from routine methodology, in which the red dots are TOC experiment results from mud log; the $7^{\text {th }}$ track is mineralogy derived from LithoScanner, including clay, quartz, nafeldspar, k-feldspar, calcite, dolomite and pyrite; the $8^{\text {th }}$ track to $18^{\text {th }}$ track are element outputs from LithoScanner, including silicon, aluminum, calcium, iron, gadolinium, sulfur, titanium, sodium, potassium, magnesium and manganese; in the $19^{\text {th }}$ track, the black curve is the total carbon derived from LithoScanner, while the blue curve is the in-organic carbon derived from LithoScanner; in the $20^{\text {th }}$ track, the black curve is the TOC derived from LithoScanner, and the red dots are TOC experiment results from mud log. Each mineral has its certain molecular formula. The minerology is derived from the elements measured by this tool. For example, quartz is calculated via silicon, while calcite is calculated via calcium. As is shown in the $20^{\text {th }}$ track, LithoScanner derived TOC matches well with mud $\log$ TOC, and the high vertical resolution of LithoScanner derived TOC shows its advantage over triple-combo derived TOC shown in $6^{\text {th }}$ track. 


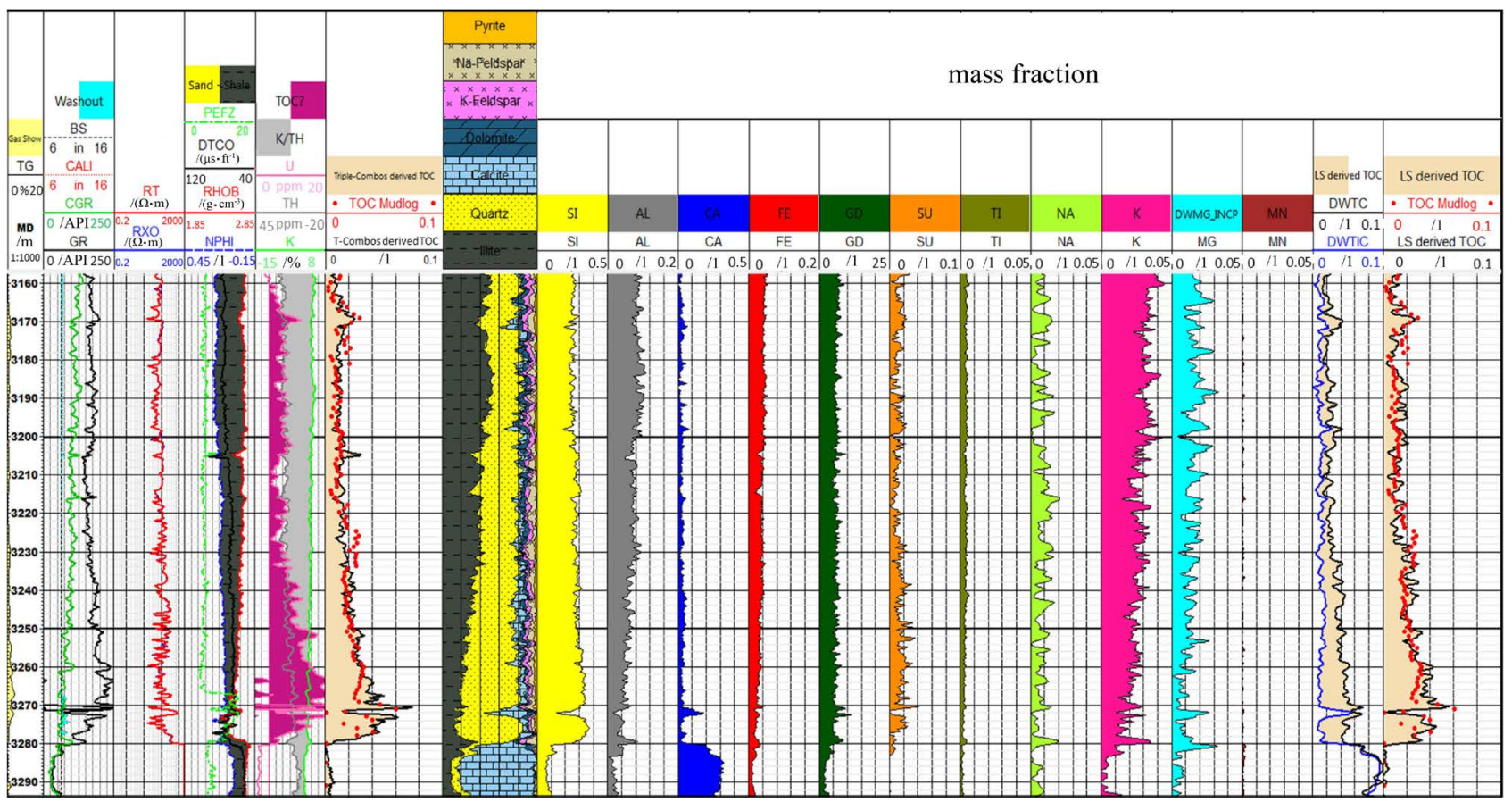

Figure 2. Triple-combo and LithoScanner interpretation result of well A.

The LithoScanner derived TOC, density derived TOC and mud log experimental TOC were compared carefully. As is shown in Figure 2, there are washouts in the borehole between $3204.3 \mathrm{~m}$ to $3205.56 \mathrm{~m}$, and $3267.5 \mathrm{~m}$ to $3278.6 \mathrm{~m}$. The density derived TOC is obviously overestimated, while the LithoScanner derived TOC has a better fit with the mud log experimental TOC. In the interval between $3271.0 \mathrm{~m}$ and $3272.8 \mathrm{~m}$, which is Wufeng-Guanyingqiao Formation, the mineralogy from LithoScanner is calcareous. Both LithoScanner derived TOC and mud log experimental TOC are low, however, the density derived TOC seems to be overestimated. The abundance of TOC in the formation is related to the eustatic sea level change. The deeper the sea level is, the greater the TOC is, vice versa. The LithoScanner derived TOC indicates the cycle changes vertically in Wufeng-Longmaxi Formation. The small vertical resolution shows the advantage of LithoScanner over triple-combos.

\subsection{Mineralogy Classification}

There are few researches on the systematical classification of lithology in shale gas reservoir, due to its complex mineralogy. A good application of LithoScanner + FMI was discussed in this paper.

LithoScanner can output accurate quartz, carbonate, and clay contents. Due to the high vertical resolution of resistivity from FMI, the mineralogy from LithoScanner can be further classified to generate sCore mineralogy output. As is shown in Figure 3, the $1^{\text {st }}$ track from the right is the sCore mineralogy output, the right plot on the top is the three-component mineralogy classification, and the right plot on the bottom is the legend.

12 mineralogies are classified in Wufeng-Longmaxi Formation (3128 - 3280 

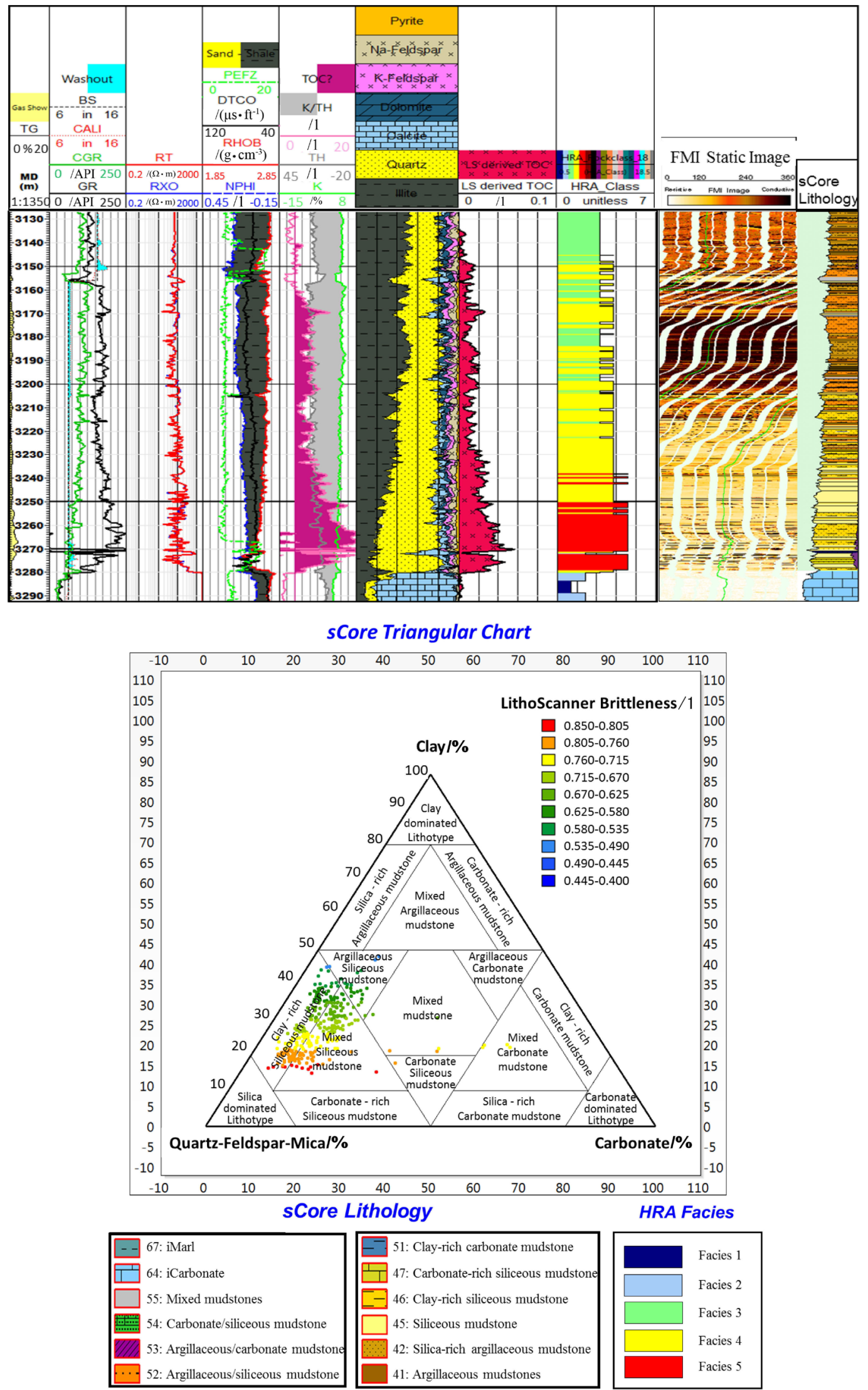

Figure 3. sCore and HRA applications using LithoScanner and FMI. 
$\mathrm{m}$ ) in well $\mathrm{A}$, and the mineralogies are dominated by silica-dominated mustone, clay-rich siliceous mudstone and silica-rich argillaceous mudstone. The mineralogies in the bottom section of Wufeng-Longmaxi Formation are dominated by silica-dominated mustone, clay-rich siliceous mudstone and silica-rich argillaceous mudstone. The dominant mineralogy gradually changes to argillaceous/ siliceous mudstone. The mineralogies in the top section of Longmaxi Formation are dominated by dark grey and grey argillaceous/siliceous mudstone and silica-rich argillaceous mudstone, interbedded with silty mudstone and thin layered siltstone. The legend of sCore is shown in the right plot at the bottom in Figure 3.

Brittleness derived from mineralogy is an important output from the sCore. The mineralogy brittleness is highly related to the completion quality in shale gas reservoirs. The more the brittle minarals are, the better the completion quality is, the more easily the formation tends to be fractured. The formula of mineralogy brittleness (MBI) derived from LithoScanner is MBI (i.e. WCAR + WQFM $) /($ WCAR + WQFM + WCLA $)$. MBI is the mineralogy brittleness; WCAR is the LithoScanner derived carbonate dry weight fraction; WQFM is the LithoScanner derived sandstone dry weight fraction, and WCLA is the LithoScanner derived clay dry weight fraction. The three-component mineralogy classification based on the mineralogy brittleness is shown in the right plot on the top in Figure 3. It shows that the more the brittle minarals are, the greater the mineralogy brittleness is. The good pay zone of Wufeng-Longmaxi Formation (3239-3280 m) of well A is plotted in the bottom left corner in the three-component plot, which indicates abundant brittle minerals, and high mineralogy brittleness.

Apart from TOC and brittleness, thermal maturity, water saturation and porosity are also necessary cutoffs for shale gas pay zone identification. In the interval of $3239-3280 \mathrm{~m}$, the Ro is around $2.4 \%-2.8 \%$, which enters the thermal maturity window for dry gas; the water saturation is below $20 \%$, and the porosity is greater than $4 \mathrm{pu}$.

In Figure 3 , the $1^{\text {st }}$ track is measured depth and total gas from mud log; the $2^{\text {nd }}$ track is GR, uranium free GR, and caliper; the $3^{\text {rd }}$ track is the deep and shallow laterolog resistivities; the $4^{\text {th }}$ track is neutron, density, DT and PEF; the $5^{\text {th }}$ track is thorium, uranium and potassium; the $6^{\text {th }}$ track is mineralogy derived from LithoScanner, including clay, quartz, na-feldspar, k-feldspar, calcite, dolomite and pyrite; the $7^{\text {th }}$ track is TOC derived from LithoScanner; the $8^{\text {th }}$ track is the HRA litho-facies result, including 5 litho-facies; the $9^{\text {th }}$ track is the static FMI resistivity image; the $10^{\text {th }}$ track is mineralogy from sCore. The three-component plot is in the left bottom corner, and the right plots at the bottom are the legend of sCore and HRA.

\subsection{Heterogeneity Rock Analysis}

Core experiments play an important role in shale gas formation evaluation. The 
vertical heterogeneity in shale formation results in low coring efficiency and difficulty in measuring the vertical heterogeneity in the reservoir. In order to improve the coring efficiency while keep a reasonable sampling rate in coring, elements dry weights from LithoScanner, such as silica, calcium, iron, sulfur, aluminum, potassium, magnesium, TOC, and triple-combos, such as GR, density, DT, resistivity were analyzed in neural network to perform an HRA analysis. Formations with similar petrophysical properites will be classified in the same group. 5 litho-facies were classified in the shale gas reservoir in well A, which can be the further guide for the coring. As is shown in Figure 3, the last track in the left plot is the litho-facies from HRA, and it can guide the coring. The legend of HRA is shown in the right corner of bottom right plot. Litho-facies type 1 is in dark blue color, while litho-facies type 2 is in shallow blue color, which are limestone in the top section of Jiancaogou Formation. Both type 1 and type 2 are characterized by low GR, high resistivity, high density, low DT, relatively low neutron and low uranium. The mineralogy from LithoScanner is dominated by limestone with low TOC. Litho-facies type 3 is in green color, which is shale in the middle-top section of Longmaxi Formation. It is characterized by medium-high GR, medium-low resistivity, high density, relatively high DT, high neutron and medium-low uranium. The mineralogy from LithoScanner is dominated by argillaceous/siliceous mudstone and silica-rich argillaceous mudstone, with relatively low TOC. Litho-facies type 4 is in yellow color, which is shale in the middle-bottom section of Longmaxi Formation. It is characterized by high GR, medium-low resistivity, relatively high density, relatively high $\mathrm{DT}$, relatively high neutron and high uranium. The mineralogy from LithoScanner is dominated by silica-dominated mudstone and clay-rich siliceous mudstone, with relatively high TOC. Litho-facies type 5 is in red color, which is shale in the bottom section of Longmaxi Formation and Wufeng Formation. It is characterized by high GR, medium-low density, high DT, medium-high neutron and high uranium. The mineralogy from LithoScanner is dominated by silica-dominated mudstone and carbonate/siliceous mudstone, with high TOC.

\subsection{Further Discussion}

1) LithoScanner derived TOC is the sum of carbon in kerogen and carbon in gas. The coefficient of volume compressibility of gas underground is very high, so the contribution of carbon from gas to the total carbon measured by the tool can not be neglected. The TOC tends to be overestimated, since the contribution of carbon from gas is not removed.

2) Due to the long period for core experiments, petrophysical derived TOC is compared with mud log experimental TOC. There might be some uncertainties.

\section{Conclusions}

New sources and detectors are equipped in LithoScanner, which enables the 
simultaneous measurements of both capture and inelastic gamma ray spectra. It is a breakthrough in spectroscopy technology. The LithoScanner derived TOC is not affected by the borehole rugosity, which improves the accuracy in TOC estimation. Systematical classification of mineralogy using LithoScanner + FMI is done, and the sCore methodology is quite robust and handy. HRA analysis is an integration of element dry weights and triple-combos, which can act as a guide for coring, and improve the coring efficiency.

Except for the carbon in carbonate and kerogen, the carbon in gas will have an impact on the uncertainty of petrophysical analysis. More works on sedimentary facies study based on the elements measured by LithoScanner can be done in the future.

\section{Funding}

This research work was supported by the Ministry of science and technology of China Petroleum \& Chemical Corporation (No. P13129).

\section{References}

[1] Charsky, A. and Herron, S. (2013) Accurate, Directtotal Organiccarbon (TOC) Log from a New Advanced Geochemical Spectroscopy Tool Comparison with Conventional Approaches for TOC Estimation. AAPG 1547013 Presented at the AAPG Annual Conference and Exhibition, Pittsburgh, 20-22 May 2013.

[2] Grau, J. and Schweitzer, J.S. (1989) Elemental Concentrations from Thermal Neutron Capture Gamma Ray Spectra in Geological Formations. Nuclear Geophysics, 3, 1-9.

[3] Herron, M.M. (1986) Mineralogy from Geochemical Well Logging. Clays and Clay Minerals, 34, 204-213. https://doi.org/10.1346/CCMN.1986.0340211

[4] Herron, S.L. and Herron, M.M. (2000) Application of Nuclear Spectroscopy Logs to the Derivation of Formation Matrix Density. Transactions of the SPWLA 41 st Annual Logging Symposium, 4-7 June 2000, Dallas.

[5] Grau, J.A., Schweitzer, J.S., Ellis, D.V. and Hertzog, R.C. (1989) A Geological Model for Gamma-Ray Spectroscopy Logging Measurements. Nuclear Geophysics, 3, 351-359.

[6] Guo, T.L. and Zhang, H.R. (2014) Formation and Enrichment Mode of Jiaoshiba Shale Gas Field, Sichuan Basin. Petroleum Exploration and Development, 41, 28-36.

[7] Guo, X.S., Li, Y.P., Liu, R.B., et al. (2014) Characteristics and Controlling Factors of Micro-Pore Structure of Longmaxi Shale Play in the Jiaoshiba Area, Sichuan Basin. Natural Gas Industry, 34, 9-16.

[8] Wei, Z.H. and Wei, X.F. (2014) Comparison of Gas-Bearing Property between Different Pore Types of Shale: A Case from the Upper Ordovician Wufeng and Longmaxi Fms in the Jiaoshiba Area, Sichuan Basin. Natural Gas Industry, 34, 37-41.

[9] Liang, C., Jiang, Z.C., Yang, Y.T., et al. (2012) Characteristics of Shale Lithofacies and Reservoir Pace of the Wufeng-Longmaxi Formation, Sichuan Basin. Petroleum Exploration and Development, 39, 691-698.

[10] Zhang, J.C., Nie, H.K., Xu, B., et al. (2008) Geological Conditions of Shale Gas Accumulation in Sichuan Basin. Natural Gas Industry, 28, 151-156.

[11] Schmoker, J. (1979) Determination of Organic Content of Appalachian Devonian 
Shales from Formation-Density Logs. American Association of Petroleum Geologists Bulletin, 63, 1504-1537.

[12] Yu, D.G., Zhao, Z.A. and Wang, Y. (2013) New Integrated Method for Petrophysical Evaluation of Shale Gas: A Case Study in Weiyuan Field. SPE 167061, SPE Unconventional Resources Conference and Exhibition-Asia Pacific, 11-13 November 2013, Brisbane.

[13] Herron, M.M., Grau, J.A., Herron, S.L., Kleinberg, R.L., Machlus, M., Reeder, S.L., Vissapragada, B., Burnham, A.K., Day, R.L. and Allix, P. (2011) Total Organic Carbon and Formation Evaluation with Wireline Logs in the Green River Oil Shale. SPE 147184, SPE Annual Technical Conference and Exhibition, 30 October-2 November 2011, Denver. https://doi.org/10.2118/147184-MS

[14] Gonzalez, J., Lewis, R., Hemingway, J., et al. (2013) Determination of Formation Organic Carbon Content Using a New Neutron-Induced Gamma Ray Spectroscopy Service That Directly Measures Carbon. SPWLA 54th Annual Logging Symposium, New Orleans, 22-26 June 2013. https://doi.org/10.1190/urtec2013-112

[15] Freedman, R., Herron, S., Anand, V., Herron, M., May, D. and Rose, D. (2014) New Method for Determining Mineralogy and Matrix Properties from Elemental Chemistry Measured by Gamma Ray Spectroscopy Logging Tools. SPE 170722, SPE Annual Technical Conference and Exhibition, 27-29 October 2014, Amsterdam. 